\title{
Thrombektomie zusätzlich zur systemischen Lyse verbessert den Outcome
}

Fragestellung: Ist die mechanische Thrombektomie in Kombination mit systemischer Lyse einer alleinigen Lyse beim akuten ischämischen Insult überlegen?

Hintergrund: Seit vielen Jahren wird beim akuten ischämischen Schlaganfall die systemische Thrombolyse mit rt-PA (Alteplase) in einem 4,5-Stunden-Zeitfenster eingesetzt. Bei Verschlüssen der großen Arterien wie beispielsweise der distalen A. carotis interna oder der proximalen $A$. cerebri media beträgt die Rekanalisierungsrate mit systemischer Thrombolyse allerdings nur etwa $50 \%$. Daher wurden in den letzten Jahren fünf große randomisierte Studien zur Thrombektomie mit einem StentRetriever bei diesen Patienten durchgeführt. Alle fünf Studien waren positiv und die Metaanalyse zeigt eine sehr hohe Rekanalisationsrate von 70 - $90 \%$ mit einer deutlichen Verbesserung des funktionellen Outcomes [1].

Die THRACE-Studie (Trial and Cost Effectiveness Evaluation of Intra-arterial Thrombectomy in Acute Ischemic Stroke) war das französische Pendant zu den anderen randomisierten Studien zur Thrombektomie.

Bracard S, Ducrocq X, Mas JL et al. Mechanical thrombectomy after intravenous alteplase versus alteplase alone after stroke (THRACE): a randomised controlled trial. Lancet Neurol 2016; 15: $1138-47$
Patienten und Methodik: Es handelte sich um eine randomisierte Studie in 26 Schlaganfallzentren in Frankreich. Eingeschlossen wurden $\mathrm{Pa}$ tienten mit akuten ischämischen Insult und Verschlüs- sen der A. carotis interna oder des proximalen Abschnitts der A. cerebri media. Die Patienten erhielten entweder eine alleinige systemische Thrombolyse oder die Kombination einer mechanischen Thrombektomie und einer systemischen Lyse. Die Thrombolyse musste innerhalb von 4 Stunden und die Thrombektomie innerhalb von 5 Stunden beginnen. Die Gefäßverschlüsse wurden durch CT oder MR-Angiografie nachgewiesen. Der primäre Endpunkt war der Prozentsatz der Patienten, der nach drei Monaten einen guten Wert auf der modifizierten Rankin-Skala von 0 bis 2 erreichte. In der Sicherheitsanalyse wurden Blutungskomplikationen und Todesfälle erfasst.

Ergebnisse: Von Juni 2010 bis Februar 2015 wurden 414 Patienten randomisiert. 208 erhielten eine alleinige Thrombolyse und 204 die Kombinationstherapie mit Thrombektomie. Drei Monate nach der Behandlung erreichten 85 der 202 Patienten (42\%) der Thrombolysegruppe einen guten Outcome, verglichen mit 106 von 200 Patienten in der Thrombektomiegruppe (53\%). Dies entspricht einer Odds Ratio von 1,55, die signifikant war. Bezüglich der Sterblichkeit mit $12 \%$ und symptomatischer intrakranieller Blutungen von $2 \%$ ergaben sich keine Unterschiede. Die Ergebnisse waren für alle Untergruppenanalysen vergleichbar.

Schlussfolgerungen: Eine Thrombektomie in Kombination mit systemischer Thrombolyse verbessert den Outcome, verglichen mit einer alleinigen systemischen Thrombolyse bei Patienten mit Verschlüssen der distalen A. carotis interna und der proximalen A. cerebri media.

\section{- Kommentar von Hans-Christoph Diener, Essen}

\section{Konzept der Kombination von Thrombektomie und Lyse bestätigt}

Die französische Studie bestätigt im Prinzip das Konzept der kombinierten Thrombektomie mit rt-PA bei Patienten mit

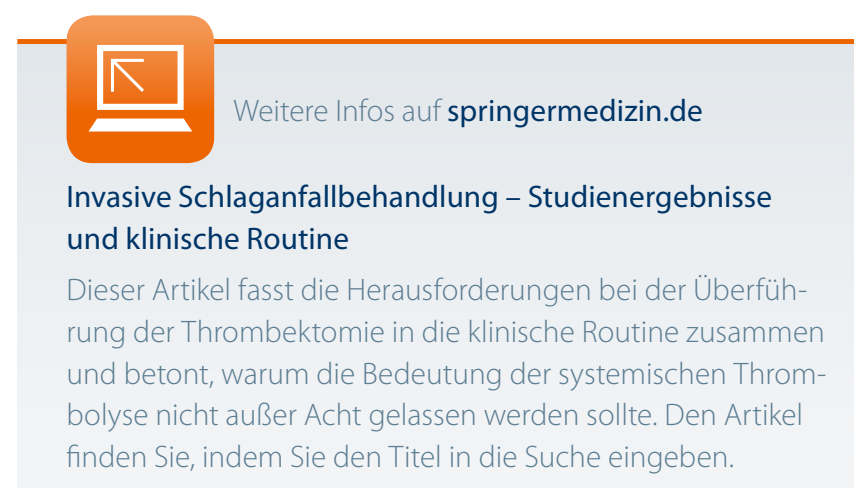

schweren Schlaganfällen und entsprechenden Gefäßverschlüssen. Der Therapieeffekt war allerdings deutlich geringer als bei vier der anderen Studien. Dies lag wahrscheinlich daran, dass der Zeitpunkt zwischen Schlaganfall und Thrombektomie länger war als in den Vergleichsstudien. Ein weiterer Schwachpunkt der Studie war sicher die lange Rekrutierung über fünf Jahre hinweg.

Negativ muss bemerkt werden, dass die Studie zu einem Zeitpunkt fortgeführt wurde, als die Ergebnisse der anderen Studien bereits bekannt waren. Die positive Konsequenz war allerdings, dass genügend Patienten behandelt wurden, um einen signifikanten Unterschied zwischen den beiden Therapiegruppen zu erreichen.

Literatur

1. Goyal M et al. Lancet 2016; 387: 1723-31 